\title{
Editorial: Pathophysiological Interrelationship Between Obesity, Metabolic Diseases, and Cancer
}

\author{
Manuel D. Gahete ${ }^{1,2,3,4 *}$, Riccarda Granata ${ }^{5}$ and Raúl M. Luque ${ }^{1,2,3,4}$ \\ ${ }^{1}$ Maimónides Institute of Biomedical Research of Córdoba (IMIBIC), Córdoba, Spain, ${ }^{2}$ Department of Cell Biology, \\ Physiology and Immunology, University of Córdoba, Córdoba, Spain, ${ }^{3}$ Reina Sofía University Hospital, Córdoba, Spain, \\ ${ }^{4}$ CIBER Pathophysiology of Obesity and Nutrition (CIBERobn), Córdoba, Spain, ${ }^{5}$ Division of Endocrinology, Diabetes and \\ Metabolism, Department of Medical Sciences, University of Turin, Turin, Italy
}

Keywords: obesity, cancer, metabolic syndrome, tumor, metabolism

Editorial on the Research Topic

Pathophysiological Interrelationship Between Obesity, Metabolic Diseases, and Cancer

\section{OPEN ACCESS}

Edited by:

Dana Kristiansson,

Norwegian Institute of Public Health

(NIPH), Norway

Reviewed by:

Alexander E. Berezin,

Zaporizhia State Medical University,

Ukraine

*Correspondence:

Manuel D. Gahete

bc2gaorm@uco.es

Specialty section:

This article was submitted to

Cancer Epidemiology and Prevention,

a section of the journal

Frontiers in Oncology

Received: 09 August 2021

Accepted: 12 August 2021

Published: 14 September 2021

Citation:

Gahete MD, Granata R and Luque RM (2021) Editorial: Pathophysiological Interrelationship Between Obesity,

Metabolic Diseases, and Cancer.

Front. Oncol. 11:755735.

doi: 10.3389/fonc.2021.755735
Obesity and metabolic syndrome are chronic endocrine-metabolic diseases that represent emerging global epidemics and capital health problems for the global population (1-3). Unfortunately, the presence of obesity and metabolic syndrome increases the risk of developing more severe endocrine pathologies [e.g. insulin resistance or type-2 diabetes (T2D)], cardiovascular complications, and some cancer types (4-8). Indeed, emerging evidence indicates that cancer development, progression, and aggressiveness are strongly influenced by the presence of metabolic alterations such as obesity and metabolic syndrome, wherein $20 \%$ of cancer incidence seems to be attributable to obesity (9). However, the precise association of the different indicators of metabolic dysregulation (body weight, obesity, metabolic syndrome, hyperglycemia, etc.), as well as the molecular, cellular, and endocrine-metabolic mechanisms underlying the pathophysiological association between metabolic abnormalities and cancer, remain to be fully elucidated.

This Research Topic compiles eight original research, clinical trial, review, and systematic review articles that advance our knowledge of and shed light on the complex pathophysiological association between obesity, metabolic diseases, and cancer. Firstly, the review by Scully et al. provides a detailed summary of the epidemiological evidence linking the different components of obesity and metabolic syndrome and their associated comorbidities (BMI, insulin resistance, hyperinsulinemia, dyslipidemia, and T2D) and different cancer types, including colon, breast, prostate, and liver. These epidemiological data are in line with those reviewed by the International Agency for Research on Cancer (IARC) and the World Cancer Research Fund/American Institute for Cancer Research (WCRF/AICR) that indicated an association of excessive body fat with increased risk of certain cancer types $(10,11)$. The review by Scully et al. recapitulates evidence from preclinical and crosssectional clinical studies, suggesting a substantial interaction between adipose tissue and cancer, which could contribute to the obesity-associated promotion of tumor growth. This relationship is especially evident in some cancer types such as breast cancer and esophageal adenocarcinoma, which are particularly affected by the obesogenic status. Examples of this close association are presented in the mini review of Bhardwaj and Brown, which explores the role of adipose tissue in obesity as a driver of breast cancer growth and development, and the review by Elliot and Reynolds, that recapitulates the epidemiological evidence supporting the association between visceral obesity and metabolic syndrome with esophageal adenocarcinoma. Moreover, the original research article 
by Ardesch et al. demonstrates the importance of dissecting out the different components of the metabolic dysregulations and the diverse indicators of obesity (BMI, adiposity, etc.) to appropriately analyze their association with cancer incidence as they demonstrate that, in the case of lung cancer, there is a nonlinear association between BMI and the risk of lung cancer while waist circumference, waist-to-hip ratio, and other body shape indexes are positively and linearly associated with the risk of this cancer type.

Therefore, the risk of cancer development in patients with obesity and/or other metabolic alterations should be specially monitored by more proactive screening programs or by implementing prevention/reversal strategies aimed to reduce the cases of different cancer types associated with metabolic dysregulations. As demonstrated by the clinical trial article by Sung et al., subjects who were willing to undergo colorectal cancer screening tended to accept a subsequent breast and prostate cancer screening, indicating the effectiveness and compliance of a onestop service for cancer screening among asymptomatic subjects, which could be specially relevant in patients with metabolic complications. In this line, the reviews by Bhardwaj and Brown and Elliot and Reynolds also recapitulate current evidence indicating the impact of obesity-related strategies, such as pharmacological interventions, physical activity, and weight lost in cancer incidence and/or development. However, these results should be interpreted considering the metabolic status of the patients, in that the systematic review by Lin et al., indicated that, in patients with T2D, weight change achieved by hypoglycemic agents or strategies over short and medium periods are not associated with incidence of most neoplasms, although it was effective in decreasing the incidence of prostate, bladder, and uterine neoplasms.

Finally, the review articles by Scully et al., Bhardwaj and Brown, and Elliot and Reynolds also examine the mechanisms underlying the pathophysiological relationship between obesity, metabolic diseases, and cancer. Among the proposed drivers of this association, they reviewed the implication of key hormonal systems (insulin/IGF-I, estrogens, or sex steroids), as well as certain adipokines, circulating lipids, and other adipose tissuesderived mediators, such as immune cells (macrophages) and cytokines/interleukins. The accumulated evidence assigning a

\section{REFERENCES}

1. Bentham J, Di Cesare M, Bilano V, Bixby H, Zhou B, Stevens GA, et al. Worldwide Trends in Body-Mass Index, Underweight, Overweight, and Obesity From 1975 to 2016: A Pooled Analysis of 2416 PopulationBased Measurement Studies in 128.9 Million Children, Adolescents, and Adults. Lancet (2017) 390:2627-42. doi: 10.1016/S0140-6736(17) 32129-3

2. Di Cesare M, Bentham J, Stevens GA, Zhou B, Danaei G, Lu Y, et al. Trends in Adult Body-Mass Index in 200 Countries From 1975 to 2014: A Pooled Analysis of 1698 Population-Based Measurement Studies With 19.2 Million Participants. Lancet (2016) 387:1377-96. doi: 10.1016/S0140-6736(16) 30054-X

3. Ng M, Fleming T, Robinson M, Thomson B, Graetz N, Margono C, et al. Global, Regional, and National Prevalence of Overweight and Obesity in Children and Adults During 1980-2013: A Systematic Analysis for the Global key role to novel mechanisms related to tumor and adipose tissues microenvironment or to the putative role of the microbiome in this pathophysiological interrelationship is also emphasized. Altogether, it seems evident the existence of common drivers that could be mediating the pathological crosstalk between obesity/adiposity/metabolic syndrome and different cancer types, as clearly illustrated by the review of $\mathrm{Ku}$ and Cheng, focused on the impact of the master regulator Activating Transcription Factor 3 (ATF3) in metabolism and cancer. ATF3 is a stress-induced transcription factor that exerts pivotal roles in modulating immune homeostasis and glucose and adipose tissue regulation, as well as shared actions on cell proliferation and metastasis in breast, prostate, colon, lung, and liver cancers. In the last study of this Research Topic, Frugé et al. integrates most of the aspect mentioned above, including the relationship between obesity and prostate cancer, weight lost interventions, microbiota, lipid profile, and changes in classic and novel molecular markers to demonstrate a multifarious association that should be further explored in future studies to finely unveil this pathological crosstalk.

Altogether, the articles included in this Research Topic add novel evidence and further support to the multifaceted pathophysiological interrelationship between obesity, metabolic diseases, and cancer. However, these articles also suggest that this is not a universal and linear relationship and should instead be analyzed by taking into account multiple parameters and considering the type of cancer, the different metabolic complications of the patients, and other confounding factors (age, race, sex, etc.). Similarly, it is also evident that the molecular, cellular, and endocrine-metabolic mechanisms that trigger the pathophysiological association between metabolic dysregulations and cancer are not fully elucidated and need further investigation.

\section{AUTHOR CONTRIBUTIONS}

MG, RG, and RL contributed to conception of the Research Topic and the Editorial. MG wrote the first draft of the Editorial. $\mathrm{RC}$ and RL revised and completed the Editorial. All authors contributed to the article and approved the submitted version.
Burden of Disease Study 2013. Lancet (2014) 384:766-81. doi: 10.1016/S01406736(14)60460-8

4. Bhaskaran K, Douglas I, Forbes H, dos-Santos-Silva I, Leon DA, Smeeth L. Body-Mass Index and Risk of 22 Specific Cancers: A Population-Based Cohort Study of 5.24 Million UK Adults. Lancet (2014) 384:755-65. doi: 10.1016/S0140-6736(14)60892-8

5. Renehan AG, Tyson M, Egger M, Heller RF, Zwahlen M. Body-Mass Index and Incidence of Cancer: A Systematic Review and Meta-Analysis of Prospective Observational Studies. Lancet (2008) 371:569-78. doi: 10.1016/ S0140-6736(08)60269-X

6. Parr CL, Batty GD, Lam TH, Barzi F, Fang X, Ho SC, et al. Body-Mass Index and Cancer Mortality in the Asia-Pacific Cohort Studies Collaboration: Pooled Analyses of 424519 Participants. Lancet Oncol (2010) 11:741-52. doi: 10.1016/S1470-2045(10)70141-8

7. Mudgway R, Chavez de Paz Villanueva C, Lin AC, Senthil M, Garberoglio CA, Lum SS. The Impact of Primary Tumor Surgery on Survival in HER2 Positive 
Stage IV Breast Cancer Patients in the Current Era of Targeted Therapy. Ann Surg Oncol (2020) 27:2711-20. doi: 10.1245/s10434-020-08310-2

8. Luque RM, López-Sánchez LM, Villa-Osaba A, Luque IM, Santos-Romero AL, Yubero-Serrano EM, et al. Breast Cancer is Associated to Impaired Glucose/Insulin Homeostasis in Premenopausal Obese/Overweight Patients. Oncotarget (2017) 8:81462-74. doi: 10.18632/oncotarget.20399

9. Arnold M, Pandeya N, Byrnes G, Renehan AG, Stevens GA, Ezzati M, et al. Global Burden of Cancer Attributable to High Body-Mass Index in 2012: A Population-Based Study. Lancet Oncol (2015) 16:36-46. doi: 10.1016/S14702045(14)71123-4

10. Lauby-Secretan B, Scoccianti C, Loomis D, Grosse Y, Bianchini F, Straif K. Body Fatness and Cancer - Viewpoint of the IARC Working Group. N Engl J Med (2016) 375:794-8. doi: 10.1056/nejmsr1606602

11. López-Suárez A. Burden of Cancer Attributable to Obesity, Type 2 Diabetes and Associated Risk Factors. Metabolism (2019) 92:136-46. doi: 10.1016/ j.metabol.2018.10.013
Conflict of Interest: The authors declare that the research was conducted in the absence of any commercial or financial relationships that could be construed as a potential conflict of interest.

Publisher's Note: All claims expressed in this article are solely those of the authors and do not necessarily represent those of their affiliated organizations, or those of the publisher, the editors and the reviewers. Any product that may be evaluated in this article, or claim that may be made by its manufacturer, is not guaranteed or endorsed by the publisher.

Copyright (c) 2021 Gahete, Granata and Luque. This is an open-access article distributed under the terms of the Creative Commons Attribution License (CC BY). The use, distribution or reproduction in other forums is permitted, provided the original author(s) and the copyright owner(s) are credited and that the original publication in this journal is cited, in accordance with accepted academic practice. No use, distribution or reproduction is permitted which does not comply with these terms. 